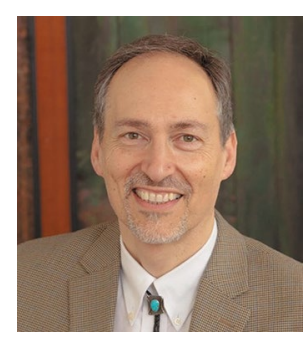

Credit: The Rockefeller

Foundation

\title{
Our food systems need inspiring and actionable vision
}

\author{
Global food systems are not fit for human and planetary health. Pessimism is easy, but does not \\ ignite positive change. This critical moment calls for visions that are optimistic, principled and \\ actionable, argues Roy Steiner.
}

$\mathrm{T}$ he COVID-19 crisis has turned long-present cracks in global food systems into fault lines, painfully and almost universally felt. At the end of 2020, hunger had more than doubled from pre-pandemic estimates, with many people experiencing food insecurity for the first time in their lives. Vulnerabilities in the supply chain led to empty grocery store shelves and food price spikes at the start of the pandemic, and inequities have been exposed as millions are pushed into poverty. What 2020 has made clear for everyone is that the global food system - from production to distribution to consumption - is not fit for the purpose of nourishing people and sustaining the health of the planet.

It can be hard to see a way out. Visions of the future in books, movies and anxiety-inducing news reports are dominated by dystopian narratives - we are getting a lot more Blade Runner, and a lot less Jetsons. But pessimistic visions don't ignite positive change, and, as much as it may be read as a cliché, we really do need to be able to visualize the world we want to leave our children.

The Rockefeller Foundation believes that positive visions can be catalytic. We believe that innovation combined with principled commitments can make the global food system more nourishing, regenerative and equitable. We have made a US $\$ 1$ billion commitment to a green, equitable COVID19 recovery, which includes long-term investments in decarbonizing food supply chains. Food is inextricably linked to the future of our climate and there is huge potential to create economic opportunity by making green energy more accessible for local agriculture. By doing this we can create food systems that not only solve multiple existing problems but more importantly give us hope for a future that we all actually want.

To begin collecting and sharing inspiring possibilities, last year we launched the Food System Vision Prize - a challenge for actionable visions on what local food systems could look like by 2050 . The entries were remarkable in their expanse of vistas and depth of thinking, and they represented the diverse, local voices - and hands that are needed to build food systems that can nourish all people and sustain the planet into the next century. We are now supporting these visionaries to bring their ideas to life.

With our broader Nourish People and Planet initiative, we are working to improve the accessibility and affordability of healthy, nutritious and sustainable diets for all. We are focused on three opportunities for innovation in this space: strengthening and greening supply networks through distributed renewable energy, transformative food policy, and leveraging the power of institutional food procurement.

\section{Strengthening and greening supply networks}

The world needs innovation to sustainably power food systems around the globe. This is essential to our climate future and the future of our food supply. As countries around the world tackle both the COVID-19 and climate crises, strengthening food chains with better infrastructure and renewable energy will be key to a sustainable and equitable recovery. We are supporting a major expansion of distributed renewable energy across Asia, Africa and Latin America. In Ethiopia, for example, we are electrifying and decarbonizing food supply chains; deploying solar pumps for irrigation; scaling up solar mini-grids for local agriculture to sustainably increase crop yields and productivity; and helping farmers reduce food loss and waste with improved logistics, cold storage and post-harvest processing. What makes this even more exciting is that with initiatives such as Landesa in Asia and sub-Saharan Africa and YieldWise in Kenya, Nigeria, Tanzania and the US, we can advocate for gender equality by ensuring women get real access to these new technologies and jobs.

\section{Transformative food policy}

Initiatives such as Finland's salt reduction programme, Denmark's whole-grain promotion and trans-fat ban, and Chile's measures on sugary beverages, show that even small, incremental changes to diet can improve health for individuals and populations. The Rockefeller Foundation is making its own push for policies that change diets and behaviour. In the US, we are helping to invest in and scale 'Food is Medicine' programmes that fully integrate nutrition into the healthcare system. This would mean that produce prescriptions and medically tailored meals, for example, could be covered through public health insurance - and high-need groups could access the healthy diets they need to live a healthy life. The compounded impact of a policy like this could equate to the reversal of currently forecasted trajectories - more than 54 million Americans with diabetes by 2030 and societal costs of more than US $\$ 622$ billion. The billions of dollars saved would be welcome reinvestment in education, circular economy infrastructure, nature conservation and research.

\section{Power of institutional food procurement}

Another strand of our work is leveraging the power of procurement at large institutions such as public schools, hospitals and universities. By supporting these institutions to optimize meals, menus, and take-home rations, we believe we can improve nutrition for millions while shaping and growing the market for healthy foods. In Rwanda, for example, The Rockefeller Foundation is in discussions with the government and local partners for a school feeding pilot in which refined, unfortified maize flour will be replaced with fortified wholegrain flour at no additional cost in select districts. In the US, we are investing in programmes that help institutions such as schools and early childhood centres set rigorous standards for the food they buy and serve to advance public health.

My outlook, even in the face of the pandemic, is optimistic. I believe that progress in nutrition security and food systems has been made and will continue to accelerate. Last year, the 
World Food Programme was awarded a Nobel Prize for feeding 100 million people in 88 countries. The sustainability of the global food system is becoming an important theme in conversations on COVID-19 recovery. In anticipation of the UN Food Systems Summit later this year, diverse stakeholders are coming together through discussion groups and virtual events to imagine what food systems of the future should look like.

At The Rockefeller Foundation, we have seen through our own imperfect history with the Green Revolution how a narrow, top-down focus on solving an urgent hunger crisis can have negative and unintended long-term consequences. The way forward - for pandemic recovery, the Food Systems Summit and the work that follows it - must focus on ensuring an equitable, inclusive approach to food systems transformation, grounded in innovative solutions and the visions of the people working at every level of our food systems.

As we look toward the UN Food Systems Summit and the global actions that will follow, The Rockefeller Foundation, in partnership with the UN Food Systems Summit Action Tracks, is supporting an inclusive search for bold solutions for our food future from every corner of the planet. And we will continue to ask, as we did the Food System Vision Prize finalists, "what is your vision for a nourishing and regenerative food future?" Because we believe that if we can imagine this together, we can build it together.

Roy Steiner ${ }^{凶}$

Senior Vice President, Food Initiative at The Rockefeller Foundation, New York, NY, USA

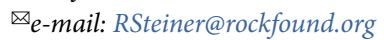

Published online: 19 March 2021

https://doi.org/10.1038/s43016-021-00246-4

Competing interests

The author declares no competing interests. 